\title{
Transient unilateral facial paralysis induced by perimesencephalic non-aneurysmal subarachnoid hemorrhage: A case report and review of the literature
}

\author{
HUI-JUN WEN, JIN-SHUO YANG and YONG-QIANG LI \\ Department of Neurology, Baoji Municipal Central Hospital, Baoji, Shanxi 721008, P.R. China
}

Received September 15, 2014; Accepted July 9, 2015

DOI: $10.3892 /$ etm.2015.2709

\begin{abstract}
The present study is, to the best of our knowledge, the first report of a rare, transient form of unilateral facial paralysis induced by perimesencephalic non-aneurysmal subarachnoid hemorrhage. The paralysis may have resulted from the compression of a part of the facial nerve by the flow of the hemorrhage into the cavum subarachnoidale; alternatively, the paralysis may have been caused by disorder of the blood supply of the facial nucleus, with the hemorrhage leading to brainstem vasospasm. The patient underwent hemostatic therapy, administration of a symptomatic antiemetic and dehydration. The facial nerve compression was released due to the absorption of the hemorrhage or the rapid improvement of the facial nucleus blood supply following the resolution of the vasospasm. Consequently, the facial nerve function was fully recovered, and the facial paralysis disappeared.
\end{abstract}

\section{Introduction}

Perimesencephalic non-aneurysmal subarachnoid hemorrhage (PNSH), identified by van Gijn and van Dongen in 1985, is a rare non-aneurysmal and benign form of subarachnoid hemorrhage (SAH) (1). The incidence of PNSH has been reported to be $8-11 \%$ in nonarteriovenous malformation and nontraumatic SAH and 21-68\% in angiogram-negative SAH (2). PNSH is characterized by a pattern of extravasated blood under computed tomography (CT) imaging, and the center of hemorrhage is located immediately anterior to the midbrain, with or without extension of blood to the basal sylvian fissure and distal interhemispheric fissure $(2,3)$. The cardinal symptoms of PNSH include headache, nausea and

Correspondence to: Dr Hui-Jun Wen, Department of Neurology, Baoji Municipal Central Hospital, 8 Jiangtan Road, Baoji, Shanxi 721008, P.R. China

E-mail: whjsq0214@163.com

Key words: perimesencephalic non-aneurysmal subarachnoid hemorrhage, facial paralysis, computed tomography, digital subtraction angiography vomiting $(2,3)$. Complications associated with PNSH include subsequent bleeding, hydrocephalus, vasospasm and recurrent hemorrhage (2). Long-term outcomes in patients with PNSH are generally improved compared with aneurysmal SAH (4). Symptoms of neurological deficit are rare, and the literature includes three cases of PNSH-induced cranial nerve palsies $(3,5,6)$. The present study describes the first case, to the best of our knowledge, of PNSH-induced transient unilateral facial paralysis.

\section{Case report}

The present case report was approved by the Ethics Committee of the Baoji Municipal Central Hospital (Baoji, China). Written informed consent was obtained from the patient. A 73-year-old male patient with a 10-year history of hypertension was admitted to the hospital presenting with headache, nausea and vomiting. His blood pressure control was optimal, and his blood pressure measured 130/80 mmHg upon admission. The neurological examination revealed no other positive signs of aphasia, hemiplegia or coma; however, the left eye of the patient closed loosely and his mouth skewed slightly to the right. Craniocerebral computed tomography (CT) provided a high-density image of a hematoma of the pontine and suprasellar cistern, suggesting SAH (Fig. 1A and B). No other lesion was observed.

On day 2 after admission, digital subtraction angiography (DSA) revealed normal cerebral blood flow, and no aneurysm or saccular projections were found (Fig. 1C-H). The patient received homostatic therapy with 6-aminocaproic acid intravenously, symptomatic antiemetic and the administration of oral nimodipine (40 $\mathrm{mg}$, three times/day) to prevent vasospasm, and was subjected to dehydration to reduce the intracranial pressure. The left-sided facial paralysis disappeared completely on day 5. Craniocerebral CT on day 8 provided an equidensity image of the pontine and suprasellar cistern, which demonstrated that the hemorrhage had been fully absorbed (Fig. 1I and J).

\section{Discussion}

The majority of cases of spontaneous SAH are caused by ruptured intracranial aneurysms. Diagnosis can usually be made through craniocerebral CT and DSA (2), although the 

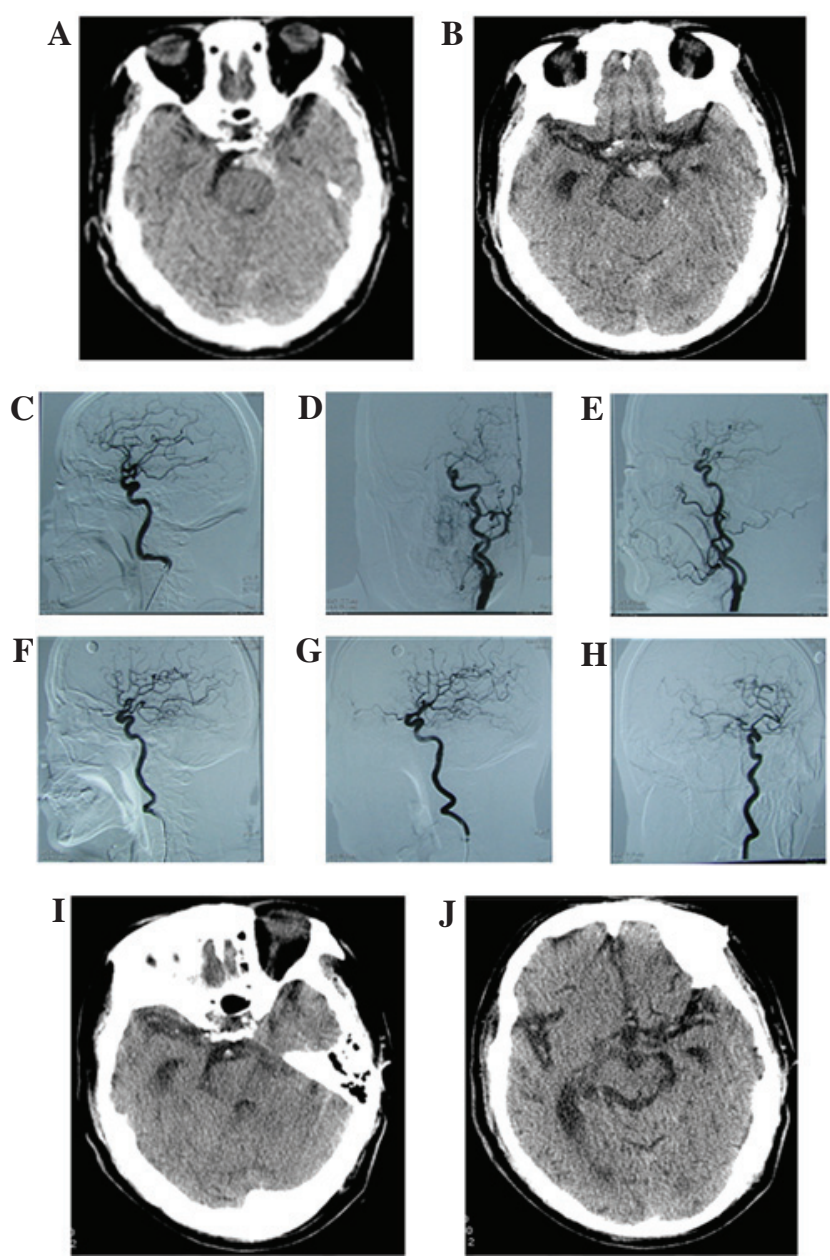

Figure 1. (A and B) Upon the admission of the patient to the hospital, craniocerebral CT provided a high-density image of a pontine and suprasellar cistern hematoma, suggesting subarachnoid hemorrhage. $(\mathrm{C}-\mathrm{H})$ On the second day after admission, digital subtract angiography examination showed normal cerebral blood flow, and no saccular projections or aneurysm were observed. (I and J) Craniocerebral CT performed on day 8 provided an equidensity image of the pontine and suprasellar cistern, which showed that the hemorrhage had been fully absorbed. CT, computed tomography.

DSA examination can give falsely negative results in $\sim 15 \%$ of patients with SAH (3). Focal neurological deficits are rare. The incidence of PNSH is low, and its presentation on CT is primarily located around the brain stem and the location is more intensive compared with other types of spontaneous SAH (4). It is generally believed that PNSH results from venous or capillary hemorrhage, with the pontine, prepontine, interpeduncular, posterior communicating or thalamic perforating veins being the most likely sources (5). In addition, PNSH is considered to be associated with vein heteromorphosis, and hypertension may also increase the risk of the disease (6).

In the present case, the initial symptoms of PNSH were headache, nausea and vomiting, which were followed by the development of complete left-sided facial paralysis by day 2 after admission. Craniocerebral CT confirmed the diagnosis of PNSH, although DSA showed no intracranial arterial abnormality, excluding the possibility of ruptured intracranial aneurysm. We believe that senile intracranial angiosclerosis resulted in decreased blood vessel elasticity and increased brittleness. At the time of attack, a sudden increase in blood pressure led to the hemorrhage, which was more concentrated near the mesencephalon. The consequent compression of the facial nerve by the hematoma, due to the limited hemorrhage area of the pontine veins (3), resulted in the complete paralysis of the left facial nerve. Alternatively, the partial SAH may have caused a vascular spasm that disturbed the blood supply of the facial nucleus, leading to left facial nerve dysfunction that manifested as complete peripheral facial paralysis.

As a result of the subsequent absorption of the hemorrhage or its continued erosion by the cerebrospinal fluid, the compression on the facial nerve was released relatively rapidly. Alternatively, the absorption of the bleeding may have resolved the partial vasospasm, allowing an improvement in the facial nucleus blood supply and restoring the function of the facial nerve. In total, the facial paralysis symptom lasted only 4 days. The craniocerebral CT scan performed on day 8 showed that the hemorrhage around the pontine had been completely absorbed, strongly confirming our speculation.

The present case illustrates that, although the main clinical symptoms of PNSH are headache, nausea and vomiting, cranial nerve dysfunction may also emerge. Three cases of oculomotor paralysis have been reported in the literature $(3,5,6)$. The first case reported a patient with PNSH that had a third cranial nerve palsy at presentation, this deficit resolved within the first week following SAH (6). The second study reported that a patient with PNSH developed 
a delayed third nerve palsy two days after the onset of SAH and this deficit was resolved by 6-month follow-up (5). The third study reported a patient with PNSH that presented as painless, pupillary-involving third cranial nerve palsy and this deficit was resolved by a 6-month follow-up (3). All these cases suggested that further studies on other cases of PNSH-induced cranial nerve palsy should be conducted.

In conclusion, the present case has demonstrated that facial paralysis can be one of the clinical manifestations of PNSH. Information regarding this symptom should be collected during clinical practice for the additional study of PNSH-induced pathologies. It is also recommended that clinicians consider the diagnosis of PNSH-induced facial paralysis as a possibility when they encounter patients presenting with transient unilateral facial paralysis combined with headache, nausea and vomiting.

\section{References}

1. van Gijn J, van Dongen KJ, Vermeulen M and Hijdra A: Perimesencephalic hemorrhage: A nonaneurysmal and benign form of subarachnoid hemorrhage. Neurology 35: 493-497, 1985

2. Schwartz TH and Solomon RA: Perimesencephalic nonaneurysmal subarachnoid hemorrhage: Review of the literature. Neurosurgery 39: 433-440; discussion 440, 1996.

3. Reynolds MR, Vega RA, Murphy RK, Miller-Thomas MM and Zipfel GJ: Perimesencephalic subarachnoid hemorrhage associated with a painless, pupillary-involving third cranial nerve palsy: Case report and literature review. Clin Neurol Neurosurg 114: 1168-1171, 2012.

4. Greebe P and Rinkel GJ: Life expectancy after perimesencephalic subarachnoid hemorrhage. Stroke 38: 1222-1224, 2007.

5. Kamat AA, Tizzard S and Mathew B: Painful third nerve palsy in a patient with perimesencephalic subarachnoid haemorrhage. Br J Neurosurg 19: 247-250, 2005.

6. Rinkel GJ, Wijdicks EF, Vermeulen M, Hasan D, Brouwers PJ and van Gijn J: The clinical course of perimesencephalic nonaneurysmal subarachnoid hemorrhage. Ann Neurol 29: 463-468, 1991. 